\title{
Category theory and consciousness
}

\author{
Goro Kato and Daniele C. Struppa \\ California Polytechnic State University, San Luis Obispo, USA / \\ George Mason University, Fairfax, USA
}

\section{Introduction}

We propose a new research program which provides an approach to consciousness and the problem of deep reality. Our model is based on category theory. The need to relate local behavior to global behavior has convinced us early on that a good model for conscious entities had to be found in the notion of sheaf. With this formulation, every presheaf represents a brain (or conscious entity). An important aspect of the theory we will develop is the notion of sheafification of a presheaf which will allow us to include complementarity as part of the description of the universe. Our model is intended to describe a notion of consciousness which is pervasive throughout the universe and not localized in individual conscious entities. At the same time, we will provide a way of describing how consciousness can arise from non-consciousness; it has always been a major problem to understand how it is possible that the fundamental components of a brain are in fact non-conscious, while the entire brain obviously possesses consciousness. In other words, we needed to provide a model which shows that "objects" which are locally trivial do not necessarily remain trivial at a global level. In our paper we do exactly this through the introduction of the new concept of consheaf (consciousness sheaf), a concept intermediate between presheaf and sheaf. The notion of consciousness has obviously been widely debated in a great variety of settings, ranging from the scientific to the philosophic. In particular, a very interesting approach to the way in which conscious identities are created was recently given by Walker Bynum (1999) in her 1999 Jefferson Lecture. Our model is in striking accordance with most of the ideas which Walker Bynum expresses from a totally different point of view, and one could see our paper as a "mathematical interpretation" of her own work. At the same time, our model is designed to be consistent with a Buddhist world-view as provided, for example, in Suzuki's and Oka's (Suzuki 1991; Oka in press). 


\section{Formulation}

In this paper, we will focus on a formulation of understanding of conscious entity. For a given moment, we see things and understand things in a restricted domain. When we get more information on the subject, we get a better picture. We would like to begin with familiar examples. When you read only three words from the third sentence on page $x$ of a piece of a literature, and only four words from the bottom sentence on page $y$, it is nearly impossible to understand the meaning of the story and the value of the literature. But if all of those parts are given to cover the entire literature, it is possible with good memory, to understand the meaning and the beauty of the literature as a complete piece. Then you will recognize each piece of information as a restriction of the whole to this particular piece of information. One can observe a similar formulation in music, personality understanding, painting, and mathematics and physics theories. It seems that a conscious entity like a human being has a constant desire to understand a global picture from the collection of locally given information.

Historically, the notion of a sheaf appeared in mathematics during 1940's by Leray in the study of topology and by Oka in the study of analytic functions of several complex variables. Then H. Cartan, Serre, and Grothendieck developed the theory of categories and sheaf cohomologies. We will use this device to study consciousness. The theory of sheaves in the categorical setting provides a general frame work for the analysis of consciousness.

Let us phrase the above mechanism in the following formulation. When an individual, whom we call $P$ tries to understand a certain global object in a category of mental activity $A$, one first observes only a part $P(U)$ of the global (whole) object. It usually requires extra time and additional information to grasp the whole of the object. Here $U$ may be considered as a part of a generalized time space $T$, i.e, propose $T$ as a model for a multi-dimensional and nonlinear time.

This generalized time space $T$ is a topological space containing $U$. One can consider the Grothendieck topology associated with the topological space $T$. Let $U$ be the category of all presheaves, i.e, all contravariant functors from $T$ to a certain product of categories indexed by a possibly uncountable set $J$.

$$
U=\left\{P: T \rightarrow \prod_{j \in I} C_{j}\right\}
$$

That is, if $P$ is an object of $U$, i.e, $P$ is a presheaf, then $P(V)=\left\{---, P_{j}(V),---\right\}$ where $P_{j}(V)$ is an object in category $C_{j}$. We suggest that $U$ is the appropriate model for a conscious universe, or as we prefer to call it, a sea of consciousness. Thoughts will be sections of such presheaves.

Suppose that these small time periods $U$ s are covering $V$, where $V$ is larger than 
$U$ in the generalized space $T$, i.e, $U \subset V$ and $V$ is maximal in the sense that for $V^{\prime}$ containing $V$, we have $P\left(V^{\prime}\right)=P(V)$ in the category $A$. If one recognizes each information $P(U)$ as the restriction of the global information $P(V)$ to $P(U)$, then we say that there exists a restriction map $\rho_{V}^{U}$ from $P(V)$ to $P(U)$ in the category $A$. This restriction map may be called the understanding of $P(U)$. Namely, $P(V)$ is a global understanding of $P(U)$. That is, for a conscious entity $P$, a formulation of an observation in category $A$, during a generalized time period $U$, is an assigned object $P(U)$ in $A$. An understanding of $P(U)$ is an object $P(V)$ in category $A$ so that there exists a restriction $\rho_{V}^{U}$ from $P(V)$ to $P(U)$ where $U \subset V$. Let us now turn to the consideration of the problem of how to model the emergence of consciousness from unconscious material. As was mentioned earlier, when a painting is looked at from very close by, the beauty of it disappears and one can claim that, locally, the painting is trivial or uninteresting. However, when all the pixels are put together, the painting acquires a totally different meaning. We therefore realized that neither just presheaves nor sheaves were the best objects to model the process of arising consciousness. What is needed is something like a sheaf in which, however, local triviality does not imply global triviality.

We therefore define the notion of consheaf as a presheaf $\mathrm{P}$ such that

1. Let $\left\{U_{j}\right\}$ be a covering for $U$, $s$ an element of $P\left(U_{j}\right)$, and $s_{j}=s_{k}$ on the intersection of $U_{j}$ and $U_{k}$; then there exists $s$ in $P(U)$ such that the restriction of $s$ to each $U_{j}$ is $s$.

2. If $s$ is an element of $P(U)$ and each restriction to the $U_{j}$ vanishes, then this does not automatically imply that $s$ is the trivial section in $P(U)$.

Note. If $P$ were a sheaf, then $s$ in condition 2 would be the trivial section in $P(U)$.

An important process in sheaf theory is the so-called sheafification process. Given a presheaf $P$, one can always construct a sheaf. (See Bredon 1997 for this construction.) The associated sheaf with this given presheaf $P$ has the same stalks of the original presheaf but not necessarily the same global sections. This associated sheaf is called the sheafificaton of the presheaf $P$. This process has an interesting interpretation in terms of the recognition of complementarity as a fundamental aspect of the universe. In fact, when a sheaf is looked upon from the point view of its stalks, its nature is particle-like and localized at each point of $T$. But if we look at a sheaf as a presheaf satisfying the sheaf axioms (namely, condition 1 and the condition in Note in the above), then its elements are sections which are distributed in nature and not at all localized. So, the same conscious entity has a double, complementary nature, which is a perfect metaphor for the particle-wave dualism of modern physics.

Next we will consider the interaction between two conscious entities. There are two different kinds of communications, which may be called vertical and horizontal communications. Let $P$ and $Q$ be two individuals, i. e. conscious entities. Through, for example, verbal or written communication methods, $P(U)$ influences $Q(U)$ in 
a category of mental activity $A$ over the generalized time period $U$. We write the communication as $P(U) \rightarrow Q(U)$.

This influence from $P$ to $Q$ over $U$ is called a morphism from $P(U)$ to $Q(U)$ in category $A$. When $U$ is not specified, this correspondence is called a natural transformation from functor $P$ to functor $Q$. This is a horizontal communication from individual $P$ to another individual $Q$.

Next, let $A$ and $B$ be two categories, e.g, mental activities. Then for an individual $P$, the thought of $P$ in the category $B$ is sometimes interpreted as a thought in a different category $A$. The diagram for the interpretation of the thought of the conscious entity $P$ in $A$ to the thought of $P$ in $B$ over the generalized time period $U$ may be written as $P_{B}(U) \rightarrow P_{A}(U)$, where $P_{B}$ and $P_{A}$ are the $B$-component and $A$-component of $P$. This communication is denoted as $f_{A B}$ from category $A$ to category $B$ so that we have $f_{A B}\left(P_{B}(U)\right)=P_{A}(U)$. This $f_{A B}$ is a functor from $A$ to $B$. This communication is what we call the vertical communication. That is the vertical communication is a personal communication. Namely, a horizontal communication is a communication between individuals in the same category, and a vertical communication is among oneself among different categories.

In her recent lecture, Walker Bynum (1999) has addressed the issue of conscious identity from a rather different viewpoint and certainly within a different intellectual framework. Her approach, too beautiful as well as too complex to be reproduced here, leads to the resolution of the body-soul dualism by defining identity as shape with a story. The body, the corporeal aspect of an identity, is identified by Walker Bynum with a shape. The shape does not carry identity unless it has a story, and this is the dynamical component of the identity. From our point of view, the body is a concrete mathematical object, and probably the best way to represent it is through the notion of set. But if we want a set to carry a story, we need to endow it with a variability through time. The perfect way of representing such a notion of variable sets is exactly through the concept of sheaf. A sheaf is a set, but not a fixed one; rather it is a set which is indexed by the family of open sets of the topological space on which it is defined. If the corporeal body is the sheaf on the topological set constituted of a single point, the shape with a story is the sheaf on $T$.

\section{Conclusions}

We have introduced a new formulation for conscious entities which follows the well know theory of categories and functors. We have interpreted several fundamental concepts in such a theory from the point of view of a theory of consciousness. In particular we have described a model for understanding and thoughts, for the process of arising of consciousness, and for communication between conscious entities and interpretation of thoughts within an individual entity. We have shown 
how our formulation is intrinsically linked to the philosophical assumption of a conscious universe and how our mathematical description is surprisingly in agreement with both Eastern and modern Western approaches to identity and consciousness. Much more work will be needed to further explore the meaning of many fundamental notions from the theory of categories. For example, we will explore whether concepts such as inverse and direct limits can shed some light onto our interpretation. We believe that what we have described indicates the naturalness of the use of category theory for the study of the formulation of consciousness.

\section{References}

Artin, M. (1962). Grothendieck topology. Mimeographed Notes, Boston: Harvard University.

Berthelot, P. and Ogus, A. (1978). Notes on crystalline cohomology. Mathematical Notes 21. Princeton: Princeton University Press.

Bredon, G.E. (1997). Sheaf theory. Berlin: Springer.

Gelfand, S. I. and Manin, Yu I. (1996). Methods of homological algebra. Berlin: Springer.

Kafatos, M. and Nadeau, R. (1990). The conscious universe. Berlin: Springer.

Lawvere, F.W. and Schanuel, S.H. (1997). Conceptual mathematics. Cambrideg: Cambridge University Press.

Mitchell, B. (1965). Theory of categories. New York: Academic Press.

Oka, K. (In press). The heart of Japan. Tokyo: Harusame Publishing.

Suzuki, D. T. (1991). The Zen doctrine of no mind. York Beach: Samuel Weiser.

Bynum, C.W. (1999). Metamorphosis in the western tradition. The 1999 Jefferson Lecture for the Humanities. http://www. neh. gov/html/bynum.html 